\title{
Stroke Unit Care Outcome in Patients with Acute Basilar Artery Occlusion Presenting Beyond Window Period
}

\author{
Dilip Maheshwari1, Vijay Sardana², Bharat Bhushan', Sankalp Mohan³, Piyush Ojha ${ }^{3}$ \\ ${ }^{1}$ Associate Professor, ${ }^{2}$ Senior Professor and Head, ${ }^{3}$ Senior Resident, \\ Deparment of Neurology, Government Medical College and Allied Hospitals, Kota, Rajasthan, India.
}

\begin{abstract}
Introduction: Posterior circulation territory stroke account for approximately one-fifth of all ischemic strokes. Basilar artery occlusion (BAO) remains a challenging diagnostic entity. BAO is associated with a high mortality rate and cases with spontaneous favorable outcomes are less frequently reported. We aim to assess the mortality and morbidity in BAO patients who presented beyond window period for thrombolytic therapy or interventional procedures.

Methods: This study was a prospective observational study conducted at a tertiary care hospital. Thirty two patients admitted to Government Medical College Kota, who presented with a clinical diagnosis of acute basilar artery occlusion supported by imaging evidence (computerized tomography, magnetic resonance imaging or angiography). Demographic details including age, sex, risk factors were recorded for all the patients along with presenting symptoms and clinical signs with radiological correlation. Functional outcome was compared between two groups, namely mechanically ventilated and nonventilated patients. Functional outcome was assessed using modified Ranking scale (mRS) on admission and at the end of 1 month follow up.
\end{abstract}

Results: Total 32 patients were enrolled during the study, which included 17 males and 15 females. Mean age of the enrolled patients was 54.14 years (range 33-67 years). Most common prodromal symptoms were vertigo and headache. Fourteen patients $(43.7 \%)$ were comatose at the onset of symptoms, $4(12.5 \%)$ patients became comatose within $48 \mathrm{hrs}$ of onset and one patient was in locked in state. Cranial nerve abnormalities and Cerebellar signs were observed in 12 and 8 patients respectively. Visual symptoms were observed in 4 patients in the form of either homonymous hemianopia or cortical blindness. Forty six percent patients in the mechanically ventilated group and $64 \%$ in the non-ventilated group were hypertensive. Out of all the patients, approximately

\section{INTRODUCTION}

Basilar artery occlusion (BAO) is an uncommon cause of stroke generally associated with a high mortality rate and a poor functional outcome in survivors. ${ }^{1,2}$ Even with thrombolytic therapy, the survival rate in BAO is only approximately $50 \%{ }^{3}$ Although many promising and approved therapies are available for anterior circulation strokes, limited options are available in cases of posterior circulation strokes to reduce morbidity and mortality. 4,5

Unlike the anterior circulation, the posterior circulation depends on one main artery that is the basilar artery which supplies the occipital lobes, part of the temporal lobes, the thalami, the cerebellum, and most importantly the brain stem .Without recanalization, the likelihood of independent outcome (defined as $\mathrm{mRS} 0-2$ ) is roughly $2 \%$ across a wide range of case series
$34 \%$ were diabetic and $38.75 \%$ had dyslipidemia. Out of 32 enrolled patients, 15 patients received mechanical ventilator support and 17 patients did not require it. Seven patients in the ventilation supported group while only 3 patients in the nonventilated group showed functional outcome improvement at the end of 1 month follow up. Three patients in without ventilator group deteriorated within seven days and two patients on ventilator supported group were deteriorated after 30 days and died. Five patients had no change in their mRS score at the end of 1 month follow up.

Conclusion: Prognosis of basilar artery occlusion patients, presenting beyond window period for thrombolysis or intervention, is poor with high mortality and morbidity. Study highlights the need for high index of suspicion for diagnosing basilar artery occlusion and the utility of mechanical ventilation in indicated cases, which may help for better functional outcome.

Key words: Basilar Artery Occlusion, Functional Outcome, Mechanical Ventilation, Prodrome.

\section{*Correspondence to:}

Dr. Dilip Maheshwari

135, Vivekanand Nagar,

Kota, Rajasthan, India.

Article History:

Received: 29-06-2016, Revised: 01-07-2016, Accepted: 03-07-2016

\begin{tabular}{|l|c|}
\hline \multicolumn{2}{|c|}{ Access this article online } \\
\hline Website: & Quick Response code \\
www.jimrp.com & \\
\hline DOI: & \\
10.21276/ijmrp.2016.2.4.025 & \\
\hline
\end{tabular}

applying thrombolysis either intravenously or intra-arterially ${ }^{6}$. Earlier studies and case reports suggest that survival with more benign outcomes have been reported in patients with $\mathrm{BAO}$, suggesting that the prognosis of $\mathrm{BAO}$ is more diverse than generally admitted. 7,8

Studies assessing the outcome of patients with basilar artery occlusion using a conventional treatment approach are very few and small, and also described the outcome in highly selected patients.

In this study, we aim to observe the outcomes of BAO patients presenting beyond window period. We also tried to analyse the predictors for mortality and morbidity in these patients and benefit of ventilator support in indicated cases. 


\section{MATERIALS AND METHODS}

The study was a prospective observational study of 32 patients, with age ranging from 18 to 70 years, admitted in a tertiary care hospital at Kota, Rajasthan from June 2014 to Jan 2016. Patients with a clinical suspicion of BAO supported by relevant imaging were enrolled for the study.

Detailed clinical history, hospital arrival time, risk factors were recorded for all the enrolled patients and functional status was measured by modified Rankin scale (mRS) on admission and at the end of one month follow up. All patients were subjected to stroke unit care.

Patients were treated with antiplatelets, statins, other supportive therapy, conservative and mechanical ventilator support in indicated cases. Patients were considered to have been treated conventionally if they had not received any specified treatment, or if they had been treated with antiplatelet, anticoagulation, or a combination of the two.

Our criteria to select for mechanical ventilation were centrally abnormal respiration patterns (apneustic, ataxic or Cheyne-Stoke breathing), aspiration pneumonitis and GCS less than 8 irrespective of oxygen saturation. Remaining patients were managed conservatively.

Patents were assessed at admission and at 1 month for functional outcome by using $\mathrm{mRS}$. The patients were divided into mRs $0-2$ who were either independent or having slight disability, mRS 3-4 who were moderately severe disabled and mRS 5- 6 who were severely disabled or died.

Informed consent for study was taken from patients/caregivers and permission taken from local ethical committee.

Table1: Demographics of Basilar artery occlusion (BAO) patients

\begin{tabular}{lcc} 
Demography & $\begin{array}{c}\text { Patients requiring } \\
\text { Ventilator Support (N=15) }\end{array}$ & $\begin{array}{c}\text { Patients without } \\
\text { Ventilator support (N=17) }\end{array}$ \\
\hline Mean Age (years) & 59.95 & 50.33 \\
Male: Female & $9: 6$ & $8: 9$ \\
Hypertension & $7(46 \%)$ & $11(64 \%)$ \\
Diabetes & $5(33 \%)$ & $6(35 \%)$ \\
Atrial Fibrillation & $1(6.6 \%)$ & $2(11.7 \%)$ \\
Dyslipidemia & $6(40 \%)$ & $6(35.2 \%)$ \\
Smoking & $7(46 \%)$ & $5(29.4 \%)$ \\
\hline
\end{tabular}

Fig 1: Functional Outcome in Different Groups of Patients (mRS)

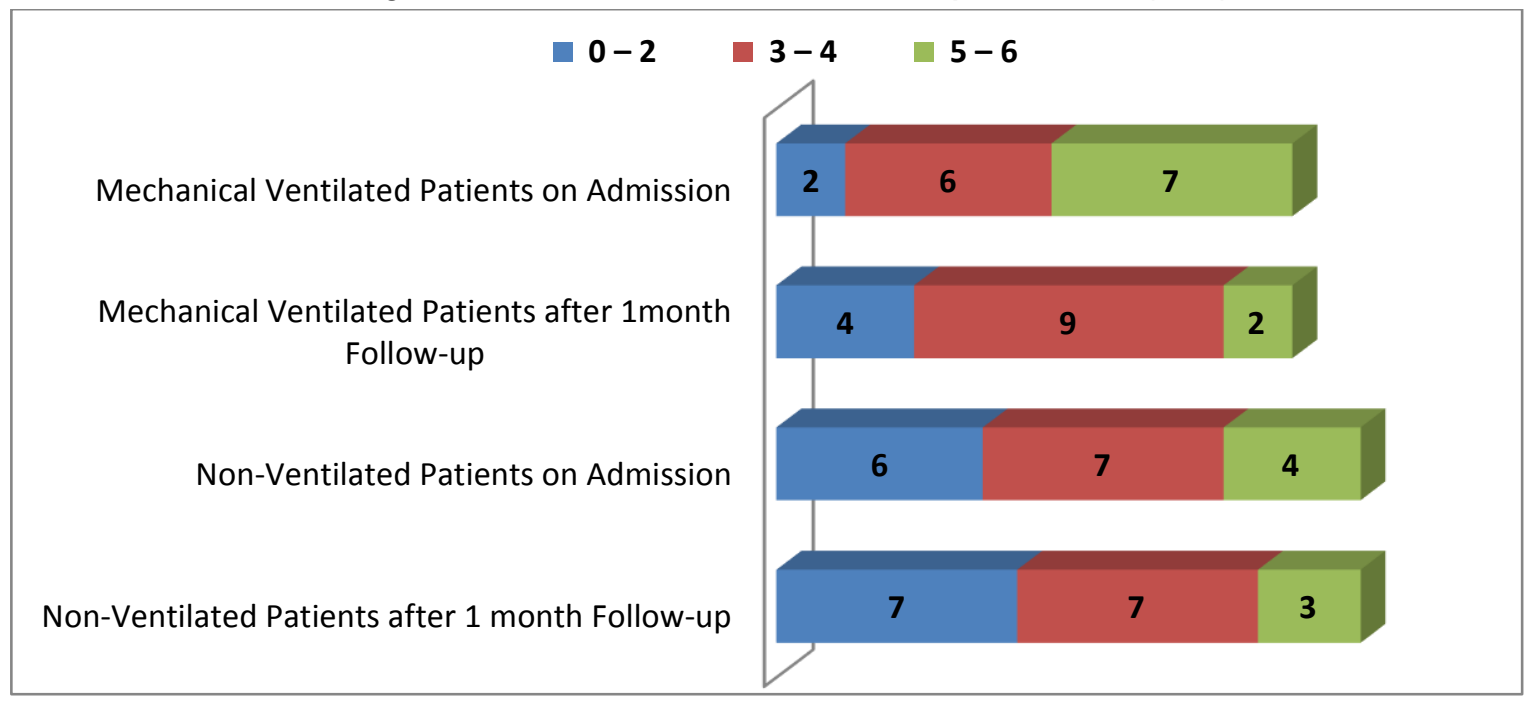

\section{RESULTS}

Demographic profile of the patients in both the group - those with ventilator support and without ventilator support is summarized in Table 1. Time taken from symptoms onset to reach the tertiary care hospital for diagnosis was ranging from 24 hours to 7 days. Most common prodromal symptoms were vertigo and headache as reported by patients and caregivers. Mean age of the patients who required ventilator support was 59.95 years and while it was 50.33 years in those who did not require mechanical ventilation. Common risk factors observed were hypertension, diabetes mellitus, smoking and dyslipidemia. Atrial fibrillation was observed in $6-12 \%$ of patients in both groups.
During follow up, patient supported with mechanical ventilator were more independent compared to those who did not require it. In both groups, total 27 patients survived at one month follow up. Seven patients showed functional outcome improvement in ventilator supported group from presentation to one month while only 3 patients showed improvement in without ventilator supported group.

Five patients who were critically ill and were enrolled in $\mathrm{mRS}$ group of 5-6 continued to remain in the same status at the end of 1 month follow up (Fig 1). Younger male patients had a better clinical outcome compared to other patients. 


\section{DISCUSSION}

Patients presenting with acute BAO beyond window period for thrombolysis or intervention have diverse clinical presentation and diagnosis is frequently delayed. BAO and resulting strokes can be devastating, resulting in severe disability or death in over $50 \%$ of cases. ${ }^{9}$ Timely diagnosis allows the use of acute interventions to recanalize the vessel, which can include intravenous thrombolysis, intra-arterial thrombolysis, and mechanical endovascular procedure. Recanalization has been shown to improve functional outcome in patients. ${ }^{10}$

The diagnosis of BAO is complicated not only by its variability in clinical presentation but also by relative infrequency with limited exposure among clinicians. The diagnosis can be significantly delayed due to absence of risk factors, low suspicion and unremarkable head CT scan. ${ }^{11}$

We are presenting demographic profile of 32 patients of BAO who presented beyond window period for thrombolytic therapy and were treated with antiplatelets, statins, other supportive measures and mechanical ventilation.

The Basilar Artery International Cooperation Study (BASICS) trial reported that their patients of radiologically confirmed BAO were between age group of 19 to 95 years. ${ }^{12}$ While most of the cases in our study were of comparatively younger age group ranging from 33 to 67 years.

Besides a dedicated stroke unit care, one reason for a favourable outcome seen in the patients in our study could be that majority of our patients belonged to younger age group. Younger patients show a better outcome with favourable mRS because the pathology involves embolic occlusion, usually top of basilar artery, a location that has been reported to have higher recanalization rates after thrombolytic therapy. ${ }^{13}$ So this is always important to specially emphasize on younger age group patients of BAO as they are expected to have better functional outcome.

In our study most common prodromal symptoms were vertigo and headache, similar to the results reported in angiographically proven cohort of 85 patients with basilar artery occlusion. ${ }^{14}$ Prodromes, minor strokes or fluctuating course have been described in several other case series also. The lesson learned from these prodromal symptoms is that the degree of suspicion should be higher and these patients should be further assessed with relevant radiological investigations to start early treatment or intervention.

In an earlier series of basilar artery occlusion patients before the era of thrombolytic therapy, the reported outcome of the patients who required mechanical ventilation was very poor with $75-90 \%$ mortality and none of the patients improved to independent functional outcome..$^{15}$ In the present study, we observed that patients who were treated in a state-of-art stroke unit care with aggressive pharmacotherapy and mechanical ventilation, in indicated cases, showed much better functional outcome.

\section{CONCLUSION}

Prodrome referable to basilar artery occlusion always needs high degree of suspicion for early relevant imaging and appropriate intervention to improve outcome in such a devastating condition. Stroke unit care and mechanical ventilation support, in indicated cases, can salvage patients and produce better functional outcome.

\section{REFERENCES}

1. Archer $C R$, Horenstein S. Basilar artery occlusion: clinical and radiological correlation. Stroke. 1977;8:383-390.

2. Caplan L. Vertebrobasilar occlusive disease. In: Barnett HJM, Mohr JP, Stein B, Yatsu F, eds. Stroke: Pathophysiology, Diagnosis and Management. New York, NY: Churchill Livingstone;1985:549-620.

3. Hacke WZeumer HFerbert ABrückmann Hdel Zoppo GJ. Intraarterial thrombolytic therapy improves outcome in patients with acute vertebrobasilar occlusive disease. Stroke.1988;19:1216-1222.

4. Hayashi J, Oguma F, Miyamura H, Eguchi S, Koike T. Direct thrombolytic revascularization of the occluded basilar artery. Cardiovasc Surg. 1993;1:547-549

5. Del Zoppo GJPessin MSMori EHacke W. Thrombolytic intervention in acute thrombotic and embolic stroke. Semin Neurol.1991;11:368-384.

6. Caplan LR. Occlusion of the vertebral or basilar artery: follow-up analysis of some patients with benign outcome. Stroke.1979;10:277282.

7. Little JRFurlan AJ. Resolving occlusive lesions of the basilar artery. Neurosurgery.1985;17:811-814.

8. Brandt TPessin MSKwan ESCaplan LR Survival with basilar artery occlusion. Cerebrovasc Dis.1995;5:182-187.

9. Mortimer AM, Bradley M, Renowden SA. Endovascular therapy for acute basilar artery occlusion: a review of the literature. J Neurointerv Surg. 2012:4:266-273.

10. Saianen T, Strbian D, Soinne L, Silvennoinen $H$, Salonen $O$, Artto V,Koskella I, Happola O, kaste M, Lindsberg PJ; Helsinki Stroke Thrombolysis Registry (HSTR) Group. Intravenous thrombolysis of basilar artery occlusion: predictors of recanalization and outcome. Stroke. 2011 Aug; 42(8):2175-9.

11. Organek N, Milano N, Donohue M, Sundararajan S, Strbian D, Katzan IL. Significant period between presentation and diagnosis in basilar artery occlusion: Five cases and lesson learned. Stroke. 2015 Apr;46(4):e79-81.

12. Greving JP, Schonewille WJ, Wijman CA, Michel P, Kappelle LJ, Algra A. BASICS Study Group. Predicting outcome after acute basilar artery occlusion based on admission characteristics. Neurology. 2012; 78:1058-1063.

13. Strbian D, Sairanen $T$, Silvennoinen $H$, Salonen $O$, Kaste $M$, Lindsberg PJ.. Thrombolysis of basilar artery occlusion: impact of baseline ischemia and time. Ann Neurol. 2013;73:688-694.

14. Ferbert A, Brückmann H, Drummen R.. Clinical features of proven basilar artery occlusion. Stroke. 1990;21:1135-1142.

15. Wijdicks EF, Scott JP. Outcome in patients with acute basilar artery occlusion requiring mechanical ventilation. Stroke. 1996 Aug; 27(8):1301-3

\section{Source of Support: Nil. Conflict of Interest: None Declared.}

Copyright: ( ) the author(s) and publisher. IJMRP is an official publication of Ibn Sina Academy of Medieval Medicine \& Sciences, registered in 2001 under Indian Trusts Act, 1882.

This is an open access article distributed under the terms of the Creative Commons Attribution Non-commercial License, which permits unrestricted non-commercial use, distribution, and reproduction in any medium, provided the original work is properly cited.

Cite this article as: Dilip Maheshwari, Vijay Sardana, Bharat Bhushan, Sankalp Mohan, Piyush Ojha. Stroke Unit Care Outcome in Patients with Acute Basilar Artery Occlusion Presenting Beyond Window Period. Int J Med Res Prof. 2016; 2(4):108-10. 